Mathematical Research Letters 3, 629-641 (1996)

\title{
THE PRIMARY APPROXIMATION TO THE COHOMOLOGY OF THE MODULI SPACE OF CURVES AND COCYCLES FOR THE STABLE CHARACTERISTIC CLASSES
}

\author{
Nariya KaWazumi And Shigeyuki Morita
}

\begin{abstract}
$\triangle$ BSTRACT. The purpose of the present note is to announce our recent results on the cohomology of the moduli space of curves or equivalently (over the rationals) the cohomology of the mapping class group of orientable surfaces.

Our main results are twofold. First we construct explicit group cocycles for any of the known stable characteristic classes (the Mumford-MoritaMiller classes) of the moduli spaces. Secondly, by combining our result with that of.Hain in [H2], we show that the "continuous part" of the cohomology of the moduli space (see $\S 5$ for the definition) is exactly equal to the subalgebra generated by the above stable classes. This second result may be considered as a supporting evidence for the conjecture that the stable cohomology of the moduli spaces would be equal to the polynomial algebra generated by the Mumford-Morita-Miller classes.

The details of the results sketched in this note will appear elsewhere.
\end{abstract}

\section{Statement of the main results}

Let $\Sigma_{g}$ be a closed oriented surface of genus $g \geq 2$ and let $\mathcal{M}_{g}$ be the mapping class group of $\Sigma_{g}$. Namely it is the group of isotopy classes of orientation preserving diffeomorphisms of $\Sigma_{g}$. Also let $\mathcal{M}_{g, *}$ and $\mathcal{M}_{g, 1}$, respectively, be the mapping class group of $\Sigma_{g}$ fixing the base point and an embedded disk $D^{2} \subset \Sigma_{g}$ pointwise. $\mathcal{M}_{g}$ acts on the Teichmüller space $\mathcal{T}_{g}$ of $\Sigma_{g}$ properly discontinuously and the quotient space $\mathbb{M}_{g}=\mathcal{T}_{g} / \mathcal{M}_{g}$ is the moduli space of genus $g$ curves. This induces a natural isomorphism $H^{*}\left(\mathbb{M}_{g} ; \mathbb{Q}\right) \cong H^{*}\left(\mathcal{M}_{g} ; \mathbb{Q}\right)$ and by virtue of a fundamental result of Harer [Ha], we can speak of the stable cohomology algebra

$$
\lim _{g \rightarrow \infty} H^{*}\left(\mathbb{M}_{g} ; \mathbb{Q}\right) \cong \lim _{g \rightarrow \infty} H^{*}\left(\mathcal{M}_{g} ; \mathbb{Q}\right)
$$

Received July 24, 1996. 
of the moduli spaces or the mapping class groups, which can also be written as $\lim _{g \rightarrow \infty} H^{*}\left(\mathcal{M}_{g, 1} ; \mathbb{Q}\right)$. We have similar stable cohomology algebra for the moduli space $\mathbb{C}_{g}=\mathcal{T}_{g, *} / \mathcal{M}_{g, *}$ of genus $g$ curves with one distinguished point and $\mathcal{M}_{g, *}$.

In the following, we formulate our results in the context of group cohomology of the mapping class groups rather than the rational cohomology of the moduli spaces. In particular, we denote $e_{i} \in H^{2 i}\left(\mathcal{M}_{g} ; \mathbb{Z}\right)(i=1,2, \ldots)$ for the Mumford-Morita-Miller classes introduced in [Mu][Mo1]. Also we write $e \in H^{2}\left(\mathcal{M}_{g, *} ; \mathbb{Z}\right)$ for the Euler class of the central extension $\mathcal{M}_{g, 1} \rightarrow$ $\mathcal{M}_{g, *}$. These characteristic classes induce the following commutative diagram

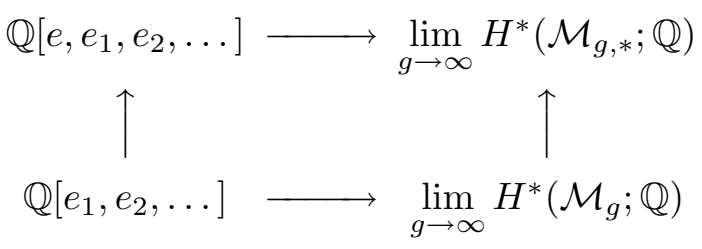

where the two horizontal homomorphisms are known to be injective by Miller [Mi] and Morita [Mo1], independently, for the lower one and by Morita [Mo1] for the upper one. It is a basic problem to determine whether these homomorphisms are surjective (and hence bijective) or not.

We write simply $H$ for the first integral homology group $H_{1}\left(\Sigma_{g} ; \mathbb{Z}\right)$ of the surface $\Sigma_{g}$. The mapping class group $\mathcal{M}_{g}$ acts on $H$ naturally and if we fix a symplectic basis of $H$, then we obtain the classical representation $\rho_{0}$ : $\mathcal{M}_{g} \rightarrow \operatorname{Sp}(2 g ; \mathbb{Z})$. By making use of natural actions of the mapping class groups on the lower central series of the fundamental groups of surfaces, in [Mo4][Mo5] the second author constructed a series of representations of the mapping class groups. The first one is nothing but the classical representation $\rho_{0}$ above and the second one can be expressed as

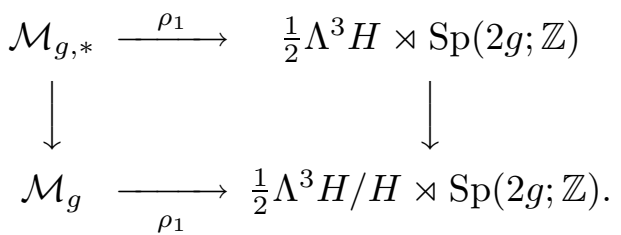

Here $\Lambda^{3} H$ denotes the third exterior power of $H$ which admits a natural action of $\operatorname{Sp}(2 g ; \mathbb{Z})$ and $H$ is considered as a submodule of $\frac{1}{2} \Lambda^{3} H$ by the embedding $H \ni x \mapsto x \wedge \omega_{0} \in \Lambda^{3} H$ where $\omega_{0} \in \Lambda^{2} H$ is the symplectic class. The representation $\rho_{1}$ is explicitly given by $\rho_{1}(\varphi)=\left(\tilde{k}(\varphi), \rho_{0}(\varphi)\right)(\varphi \in$ $\left.\mathcal{M}_{g, *}\right)$, where $\tilde{k}: \mathcal{M}_{g, *} \rightarrow \frac{1}{2} \Lambda^{3} H$ is a crossed homomorphism which extends Johnson's homomorphism of the Torelli group $\tau: \mathcal{I}_{g, *} \rightarrow \Lambda^{3} H$ given in [J1]. 
Now let $\operatorname{Hom}\left(\Lambda^{*}\left(\frac{1}{2} \Lambda^{3} H\right), \mathbb{Q}\right)^{\mathrm{Sp}}$ be the set of all $\operatorname{Sp}(2 g ; \mathbb{Z})$-invariant homomorphisms from the exterior power of the abelian group $\frac{1}{2} \Lambda^{3} H$ to $\mathbb{Q}$. It is easy to see that any element of this module can be naturally considered as cocycles of the semi-direct product $\frac{1}{2} \Lambda^{3} H \rtimes \mathrm{Sp}(2 g ; \mathbb{Z})$ (see $\S 4$ ) so that we obtain the following commutative diagram

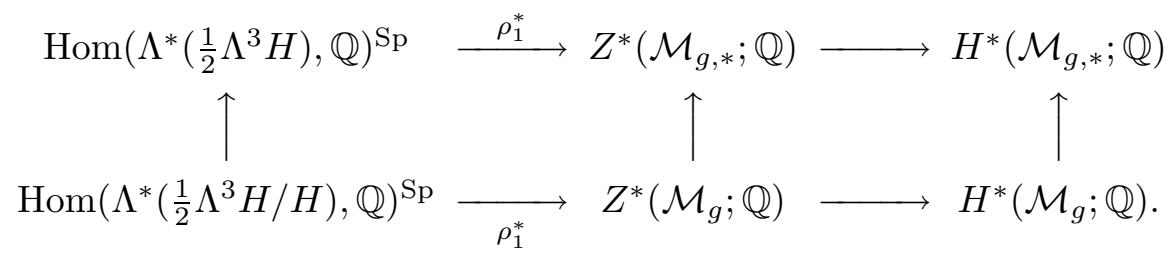

Here and throughout this note we denote by $C^{*}(G ; M)$ the normalized standard cochain complex of a group $G$ with coefficients in a $G$-module $M$ and by $Z^{*}(G ; M)$ the set of cocycles in $C^{*}(G ; M)$.

As was already shown in [Mo8], the images of the homomorphisms $\rho_{1}^{*}$ contain the Euler class $e$ and the characteristic classes $e_{i}$ for all $i \geq 1$. Our main result is

Theorem 1. The homomorphisms $\rho_{1}^{*}$ factor through the subalgebras generated by the characteristic classes $e, e_{i}(i=1,2, \ldots)$. More precisely, we have the following commutative diagram

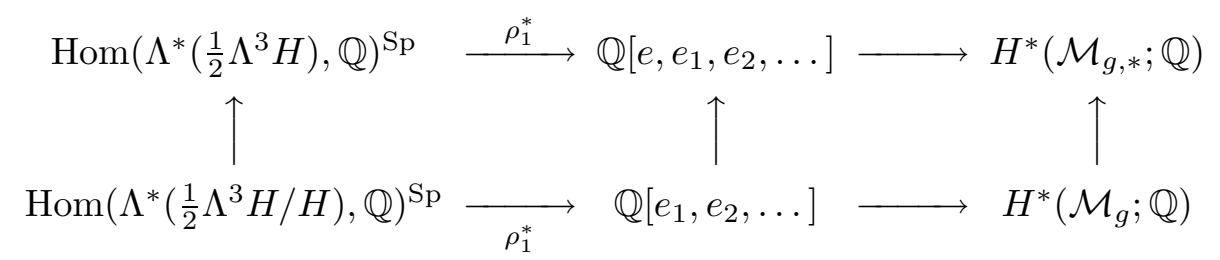

so that the images of the homomorphisms $\rho_{1}^{*}$ are exactly equal to the subalgebras generated by the Euler class and the Mumford-Morita-Miller classes. Moreover we have explicit formulae for $\rho_{1}^{*}$.

\section{The generalized Mumford-Morita-Miller classes}

Let $\overline{\mathcal{M}}_{g, *}$ be the fiber product $\mathcal{M}_{g, *} \times \mathcal{M}_{g} \mathcal{M}_{g, *}$ induced from the forgetful homomorphism $\mathcal{M}_{g, *} \rightarrow \mathcal{M}_{g}$. It is easy to see that the correspondence $\overline{\mathcal{M}}_{g, *} \ni(\varphi, \psi) \mapsto\left(\psi \varphi^{-1}, \varphi\right) \in \pi_{1} \Sigma_{g} \rtimes \mathcal{M}_{g, *}$ defines an isomorphism. As in [Mo3], let us consider a mapping

$$
k_{0}: \overline{\mathcal{M}}_{g, *} \longrightarrow H
$$

defined by $k_{0}((\varphi, \psi))=\left[\psi \varphi^{-1}\right] \in H$. Since $\psi \phi^{-1}$ is contained in the kernel of the forgetful homomorphism $\mathcal{M}_{g, *} \rightarrow \mathcal{M}_{g}$, which is naturally isomorphic 
to $\pi_{1} \Sigma_{g}$, we may consider its abelianization $\left[\psi \phi^{-1}\right] \in H$. Then it is easy to see that $k_{0}$ satisfies the cocycle condition

$$
k_{0}\left(\left(\varphi_{1}, \psi_{1}\right)\left(\varphi_{2}, \psi_{2}\right)\right)=k_{0}\left(\left(\varphi_{1}, \psi_{1}\right)\right)+\left(\varphi_{1}\right)_{*} k_{0}\left(\left(\varphi_{2}, \psi_{2}\right)\right) .
$$

Hence it can be considered as an element of $Z^{1}\left(\overline{\mathcal{M}}_{g, *} ; H\right)$. Let $\pi: \overline{\mathcal{M}}_{g, *} \rightarrow$ $\mathcal{M}_{g, *}$ and $\bar{\pi}: \overline{\mathcal{M}}_{g, *} \rightarrow \mathcal{M}_{g, *}$, respectively, be the first (resp. the second) projections so that we have the fibre square

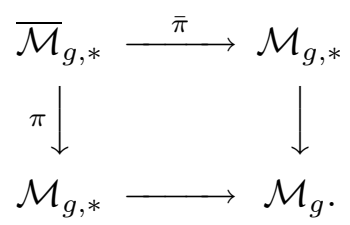

Now we write simply $\bar{e}$ for $(\bar{\pi})^{*}(e) \in H^{2}\left(\overline{\mathcal{M}}_{g, *} ; \mathbb{Z}\right)$ and we consider the cohomology class $\bar{e}^{i} k_{0}^{j} \in H^{2 i+j}\left(\overline{\mathcal{M}}_{g, *} ; \Lambda^{j} H\right)$. We define

$$
m_{i, j}=\pi_{!}\left(\bar{e}^{i} k_{0}^{j}\right) \in H^{2 i+j-2}\left(\mathcal{M}_{g, *} ; \Lambda^{j} H\right)
$$

for $i, j \geq 0$ and $i+j \geq 2$, where $\pi_{!}: H^{k}\left(\overline{\mathcal{M}}_{g, *} ; M\right) \rightarrow H^{k-2}\left(\mathcal{M}_{g, *} ; M\right)$ is the Gysin homomorphism (or the integral along the fibers of the map $\pi$ ). We call them the generalized Mumford-Morita-Miller classes. In fact, when $j=0$ and $i \geq 1$, we have $m_{i+1,0}=e_{i}$. On the mapping class group $\mathcal{M}_{g, 1}$ these classes are nothing but the cohomology classes $(-1)^{j} m_{i, j}$ introduced by the first author in [Ka1] (see also [Ka3]).

Recently Looijenga [L1] obtained a remarkable result that the rational stable cohomology of $\mathcal{M}_{g}$ with coefficients in any finite dimensional irreducible representation $M$ of the algebraic group $\operatorname{Sp}(2 g ; \mathbb{Q})$ is isomorphic to a free module over the stable rational cohomology of $\mathcal{M}_{g}$ together with a description of its free basis. His proof and construction of cohomology classes are based on geometric considerations on the moduli orbifold of complex algebraic curves including, in particular, a theorem in Hodge theory. As for $\mathcal{M}_{g}$, this result cannot be generalized to integral symplectic coefficients. In fact, for example, the second integral cohomology of $\mathcal{M}_{g}$ with coefficients in $H$ does not admit the stability [Mo2].

On the basis of Looijenga's noteworthy idea [L1] that the stable cohomology with symplectic coefficients is derived only from the Harer stability theorem [Ha] with trivial coefficients, the first author has deduced the following result: the stable integral cohomology of $\mathcal{M}_{g, 1}$ with coefficients in $H^{\otimes n}$ is a free module over the stable integral cohomology of $\mathcal{M}_{g, 1}$, and certain algebraic combinations of the (modified) generalized Mumford-MoritaMiller classes can serve as its (topologically constructed and new) free basis 
[Ka2]. Here the Lyndon-Hochschild-Serre spectral sequence for a pair of groups introduced in [Ka1] is used instead of geometric considerations including Hodge theory.

Since it has been found out that the generalized Mumford-Morita-Miller classes can be defined also on $\mathcal{M}_{g, *}$, all the results obtained in [Ka2] hold for the mapping class group $\mathcal{M}_{g, *}$. For example, we have

Theorem 2. If the total degree is smaller than $g / 2$, then we have

$$
H^{*}\left(\mathcal{M}_{g, *} ; \Lambda^{*} H \otimes \mathbb{Q}\right)=H^{*}\left(\mathcal{M}_{g, *} ; \mathbb{Q}\right) \otimes\left(\bigotimes_{i, j} \mathbb{Q}\left[m_{i, j}\right]\right)
$$

where the indices $(i, j)$ run over the set $\{i \geq 0, j \geq 1$ and $i+j \geq 2\}$.

The above results show that we have obtained explicit basis for these free modules in terms of the generalized Mumford-Morita-Miller classes defined above.

\section{Description of the Sp-invariant cocycles}

In this section, we describe the spaces of Sp-invariant alternating cocycles

$$
\operatorname{Hom}\left(\Lambda^{*}\left(\frac{1}{2} \Lambda^{3} H\right), \mathbb{Q}\right)^{\mathrm{Sp}}, \quad \operatorname{Hom}\left(\Lambda^{*}\left(\frac{1}{2} \Lambda^{3} H / H\right), \mathbb{Q}\right)^{\mathrm{Sp}}
$$

of the abelian groups $\frac{1}{2} \Lambda^{3} H, \frac{1}{2} \Lambda^{3} H / H$. For any positive integer $r$, let $\mathcal{G}_{2 r}$ denote the set of isomorphism classes of connected trivalent graphs with $2 r$ vertices and let $\mathcal{G}_{2 r}^{0}$ be the subset of $\mathcal{G}_{2 r}$ consisiting of those graphs without loops. Here a loop means an edge whose two endpoints are attached to the same vertex. We set $\mathcal{G}$ (resp. $\mathcal{G}^{0}$ ) to be the disjoint union of $\mathcal{G}_{2 r}$ (resp. $\mathcal{G}_{2 r}^{0}$ ) for all $r \geq 1$

$$
\mathcal{G}=\coprod_{r \geq 1} \mathcal{G}_{2 r}, \quad \mathcal{G}^{0}=\coprod_{r \geq 1} \mathcal{G}_{2 r}^{0}
$$

Now let

$$
\mathbb{Q}\left[\alpha_{\Gamma} ; \Gamma \in \mathcal{G}\right]
$$

be the polynomial algebra over $\mathbb{Q}$ generated by $\alpha_{\Gamma}$ for each element $\Gamma \in \mathcal{G}$. We define the degree of $\alpha_{\Gamma}\left(\Gamma \in \mathcal{G}_{2 r}\right)$ to be equal to $2 r$. We also consider a similar polynomial algebra $\mathbb{Q}\left[\beta_{\Gamma} ; \Gamma \in \mathcal{G}^{0}\right]$.

Let $\Gamma$ be a graph belonging to $\mathcal{G}_{2 r}$. We put a vector of the module $\Lambda^{3} H$ on each vertex of $\Gamma$. Each edge indicates that two vectors on its terminal vertices should be contracted by the intersection pairing $\mu: H \otimes H \rightarrow \mathbb{Z}$. 
This means that each homogeneous element of degree $2 r$ in $\mathbb{Q}\left[\alpha_{\Gamma} ; \Gamma \in \mathcal{G}\right]$ defines a way of contraction

$$
\alpha_{\Gamma}: \Lambda^{2 r}\left(\Lambda^{3} H\right) \longrightarrow \mathbb{Q}
$$

which is a linear combination of the restrictions of various iterated contractions on $H^{\otimes 6 r}$ induced from the intersection pairing $\mu$. Thus we obtain a natural homomorphism

$$
\mathbb{Q}\left[\alpha_{\Gamma} ; \Gamma \in \mathcal{G}\right] \longrightarrow \operatorname{Hom}\left(\Lambda^{*}\left(\frac{1}{2} \Lambda^{3} H\right), \mathbb{Q}\right)^{\mathrm{Sp}} .
$$

A result of Weyl in the classical representation theory implies that the above homomorphism is surjective and is an isomorphism for degrees $\leq \frac{2}{3} g$. Also let $q: \Lambda^{3} H_{\mathbb{Q}} \rightarrow \Lambda^{3} H_{\mathbb{Q}}$ be the homomorphism defined by $q(\xi)=$ $\xi-\frac{1}{2 g-2} C \xi \wedge \omega_{0}\left(\xi \in \Lambda^{3} H\right)$, where $H_{\mathbb{Q}}=H \otimes \mathbb{Q}$ and $C: \Lambda^{3} H \rightarrow H$ is the contraction. It is easy to see that $q$ is Sp-equivariant and that $q(H)=0$ so that it induces a homomorphism $\Lambda^{3} H_{\mathbb{Q}} / H_{\mathbb{Q}} \rightarrow \Lambda^{3} H_{\mathbb{Q}}$. Now we define a homomorphism

$$
\mathbb{Q}\left[\beta_{\Gamma} ; \Gamma \in \mathcal{G}^{0}\right] \longrightarrow \operatorname{Hom}\left(\Lambda^{*}\left(\frac{1}{2} \Lambda^{3} H / H\right), \mathbb{Q}\right)^{\mathrm{Sp}}
$$

by setting $\beta_{\Gamma}: \Lambda^{2 r}\left(\frac{1}{2} \Lambda^{3} H / H\right) \rightarrow \mathbb{Q}$ to be equal to the composition $\alpha_{\Gamma} \circ q$ : $\Lambda^{2 r}\left(\Lambda^{3} H_{\mathbb{Q}} / H_{\mathbb{Q}}\right) \rightarrow \Lambda^{2 r}\left(\Lambda^{3} H_{\mathbb{Q}}\right) \rightarrow \mathbb{Q}$. Weyl's result again implies that this homomorphism is also surjective and bijective in the same range as above. Moreover we can define a twisted inclusion $\mathbb{Q}\left[\beta_{\Gamma} ; \Gamma \in \mathcal{G}^{0}\right] \rightarrow \mathbb{Q}\left[\alpha_{\Gamma} ; \Gamma \in \mathcal{G}\right]$ so that all the above constructions are compatible with each other. In view of the above description, Theorem 1 follows from

Theorem 3. For any $\Gamma \in \mathcal{G}$ or $\Gamma \in \mathcal{G}^{0}$, we have

$$
\rho_{1}^{*}\left(\alpha_{\Gamma}\right) \in \mathbb{Q}\left[e, e_{1}, e_{2}, \ldots\right], \quad \rho_{1}^{*}\left(\beta_{\Gamma}\right) \in \mathbb{Q}\left[e_{1}, e_{2}, \ldots\right]
$$

which gives explicit group cocycles for any of the Mumford-Morita-Miller classes $e_{i}$.

Similar result holds for $\mathcal{M}_{g, 1}$ and it is remarkably simple. Namely we have

Theorem 4. Let $\Gamma \in \mathcal{G}_{2 i}$ be any element. Then the cocycle $\alpha_{\Gamma} \in Z^{2 i}\left(\mathcal{M}_{g, 1} ; \mathbb{Q}\right)$ represents the $i$-th Mumford-Morita-Miller class $e_{i}$ (up to signs). 


\section{Outline of the proof of the main result}

As was stated at the end of the previous section, Theorem 1 follows from Theorem 3. Here we give an outline of the proof of Theorem 3 .

In general, let $Q$ be a group, $N$ a $Q$-module and $M$ an $N \rtimes Q$-module. For an $r$-cochain $c \in C^{r}(N ; M)$ of $N$, we define its (natural) extension $\widetilde{c} \in C^{r}(N \rtimes Q ; M)$ by setting

$$
\begin{aligned}
& \widetilde{c}\left(\left(n_{1}, q_{1}\right),\left(n_{2}, q_{2}\right), \ldots,\left(n_{r}, q_{r}\right)\right)= \\
& \quad c\left(n_{1}, q_{1}\left(n_{2}\right), q_{1} q_{2}\left(n_{3}\right), \ldots, q_{1} q_{2} \cdots q_{r-1}\left(n_{r}\right)\right)
\end{aligned}
$$

for $n_{i} \in N, q_{i} \in Q$. It turns out that the restriction of this extending operation to the invariants $C^{*}(N ; M)^{Q} \ni c \mapsto \widetilde{c} \in C^{*}(N \rtimes Q ; M)$ is a cochain map. Suppose that there is given a homomorphism $\rho: G \rightarrow N \rtimes Q$ from a group $G$ to the semi-direct product $N \rtimes Q$. Then $\rho$ can be expressed as $\rho=(f, \bar{\rho})$. Here $f: G \rightarrow N$ is a 1-cocycle of $G$ with values in the $G$ module $N$ where the action is given through the homomorphism $\bar{\rho}: G \rightarrow Q$. For the trivial module $\mathbb{Q}$, we have a linear map

$$
\rho^{*}: \operatorname{Hom}\left(\Lambda^{*} N, \mathbb{Q}\right)^{Q} \hookrightarrow Z^{*}(N ; \mathbb{Q})^{Q} \rightarrow Z^{*}(N \rtimes Q ; \mathbb{Q}) \rightarrow Z^{*}(G ; \mathbb{Q}) .
$$

The cocycle $\rho^{*} c \in Z^{r}(G ; \mathbb{Q})$ induced from $c \in \operatorname{Hom}\left(\Lambda^{r} N, \mathbb{Q}\right)^{Q}$ is explicitly given by

$$
\begin{aligned}
\left(\rho^{*} c\right)\left(g_{1}, g_{2}, \ldots, g_{r}\right) & =c\left(f\left(g_{1}\right), \bar{\rho}\left(g_{1}\right) f\left(g_{2}\right), \ldots, \bar{\rho}\left(g_{1} \cdots g_{r-1}\right) f\left(g_{r}\right)\right) \\
& =\left(c_{*} f^{r}\right)\left(g_{1}, g_{2}, \ldots, g_{r}\right) \quad\left(g_{i} \in G\right),
\end{aligned}
$$

where $f^{r} \in Z^{r}\left(G ; \Lambda^{r} N\right)$ means the $r$-th power of the 1-cocycle $f$, with respect to the Alexander-Whitney cup-product, and $c_{*}: C^{*}\left(G ; \Lambda^{r} N\right) \rightarrow$ $C^{*}(G ; \mathbb{Q})$ denotes the cochain map induced from the $G$-homomorphism $c: \Lambda^{r} N \rightarrow \mathbb{Q}$. Consequently we obtain

$$
\left[\rho^{*} c\right]=c_{*}[f]^{r} \in H^{r}(G ; \mathbb{Q}) .
$$

Now we apply the above procedure to the homomorphism $\rho_{1}$ given in $\S 1$. As was stated in $\S 3$, any connected trivalent graph $\Gamma \in \mathcal{G}_{2 r}$ defines an element $\alpha_{\Gamma} \in \operatorname{Hom}\left(\Lambda^{2 r}\left(\frac{1}{2} \Lambda^{3} H\right), \mathbb{Q}\right)^{S p}$. Hence the cohomology class $\rho_{1}^{*} \alpha_{\Gamma} \in H^{2 r}\left(\mathcal{M}_{g, *} ; \mathbb{Q}\right)$ is obtained from the cohomology class $\tilde{k}^{2 r} \in$ $H^{2 r}\left(\mathcal{M}_{g, *} ; \Lambda^{2 r}\left(\frac{1}{2} \Lambda^{3} H\right)\right)$ by applying the contraction, associated with the graph $\Gamma$, on the coefficients.

Besides the introduction of the generalized Mumford-Morita-Miller classes $m_{i, j}$ described in $\S 2$, the key to the proof of Theorem 3 is 
Lemma. $m_{0,3}=\pi_{!}\left(k_{0}{ }^{3}\right)=-6 \tilde{k} \in H^{1}\left(\mathcal{M}_{g, *} ; \Lambda^{3} H\right)$.

In view of the above Lemma, it suffices to show that the generalized Mumford-Morita-Miller classes of surface bundles $m_{i, j}$ are stable under any contractions of coefficients which are derived from the intersection pairing $\mu: H \otimes H \rightarrow \mathbb{Z}$.

As was shown in [Mo2] Theorem 1.3, we have

$$
\mu_{*}\left(k_{0}{ }^{2}\right)=2 \nu-\pi^{*} e-\bar{\pi}^{*} e \in H^{2}\left(\overline{\mathcal{M}}_{g, *} ; \mathbb{Z}\right) .
$$

Here $\pi$ (resp. $\bar{\pi}): \overline{\mathcal{M}}_{g, *} \rightarrow \mathcal{M}_{g, *}$ denotes the first (resp. the second) projection as before. The class $\nu$ is the "Poincaré dual" of the image of the diagonal map $s: \mathcal{M}_{g, *} \rightarrow \overline{\mathcal{M}}_{g, *}[\mathrm{Mo} 2]$. Thus any contraction of a single generalized Mumford-Morita-Miller class is expressed by an algebraic combination of other such classes. On the other hand, let $M_{1}$ and $M_{2}$ be two $\mathcal{M}_{g, *}$-modules. Then we consider the contraction map $C:\left(M_{1} \otimes H\right) \otimes$ $\left(H \otimes M_{2}\right) \rightarrow M_{1} \otimes M_{2}$ which is given by $\xi \otimes x \otimes y \otimes \eta \mapsto \mu(x \otimes y) \xi \otimes \eta$.

Contraction formula. For any two elements $u_{i} \in H^{*}\left(\overline{\mathcal{M}}_{g, *} ; M_{i}\right)(i=$ 1,2), we have the equality

$$
\begin{aligned}
& C_{*}\left(\pi_{!}\left(u_{1} \otimes k_{0}\right) \pi_{!}\left(k_{0} \otimes u_{2}\right)\right)= \\
& \quad-\pi_{!}\left(u_{1} u_{2}\right)+s^{*}\left(u_{1}\right) \pi_{!}\left(u_{2}\right)+\pi_{!}\left(u_{1}\right) s^{*}\left(u_{2}\right)-e \pi_{!}\left(u_{1}\right) \pi_{!}\left(u_{2}\right)
\end{aligned}
$$

as an element of $H^{*}\left(\mathcal{M}_{g, *} ; M_{1} \otimes M_{2}\right)$.

This implies that any contraction of two generalized Mumford-MoritaMiller classes can be expressed by an algebraic combination of other generalized Mumford-Morita-Miller classes. Consequently the cohomology class $\rho_{1}^{*} \alpha_{\Gamma} \in H^{*}\left(\mathcal{M}_{g, *} ; \mathbb{Q}\right)$ is an algebraic combination of the classes $m_{i+1,0}=e_{i}$ and $e$.

\section{Further implications of the main results}

Here we mention further results which can be obtained by combining our main theorem with Hain's fundamental results in [H1][H2]. We have a natural filtration $\left\{\mathcal{M}_{g, 1}(k)\right\}_{k \geq 0}$ on the mapping class group $\mathcal{M}_{g, 1}$, which is induced from the lower central series of the fundamental group of $\Sigma_{g}$ $D^{2}$. Namely $\mathcal{M}_{g, 1}(k)$ is defined to be the subgroup of $\mathcal{M}_{g, 1}$ consisiting of elements which act on the $(k-1)$-st nilpotent quotient of $\pi_{1}\left(\Sigma_{g} \backslash D^{2}\right)$ trivially (see [Mo4][Mo5][Mo6] for details). We can also consider another filtration $\left\{\mathcal{M}_{g, 1}^{\prime}(k)\right\}_{k \geq 0}$ where $\mathcal{M}_{g, 1}^{\prime}(1)=\mathcal{M}_{g, 1}(1)$ is the Torelli group $\mathcal{I}_{g, 1}$ and $\left\{\mathcal{M}_{g, 1}^{\prime}(k)\right\}_{k \geq 1}$ is the lower central series of $\mathcal{I}_{g, 1}=\mathcal{M}_{g, 1}(1)$. Johnson 
[J2] proved that $\mathcal{M}_{g, 1}^{\prime}(k) \subset \mathcal{M}_{g, 1}(k)$ for all $k$. Now let us consider the direct limits

$$
\begin{aligned}
& H_{c}^{*}\left(\mathcal{M}_{g, 1} ; \mathbb{Q}\right)=\lim _{k \rightarrow \infty} H^{*}\left(\mathcal{M}_{g, 1} / \mathcal{M}_{g, 1}(k) ; \mathbb{Q}\right) \\
& H_{c^{\prime}}^{*}\left(\mathcal{M}_{g, 1} ; \mathbb{Q}\right)=\lim _{k \rightarrow \infty} H^{*}\left(\mathcal{M}_{g, 1} / \mathcal{M}_{g, 1}^{\prime}(k) ; \mathbb{Q}\right)
\end{aligned}
$$

of the cohomology of the successive quotients and, following Hain, we call them the continuous cohomology of the mapping class group. We have a natural forgetful homomorphism from the continuous cohomology of $\mathcal{M}_{g, 1}$ to the usual cohomology and its image may be called the continuous part of $H^{*}\left(\mathcal{M}_{g, 1} ; \mathbb{Q}\right)$. In these constructions, we may further pass to the limit as $g \rightarrow \infty$.

We have extensions

$$
1 \longrightarrow \mathcal{I}_{g, 1} / \mathcal{M}_{g, 1}(k) \longrightarrow \mathcal{M}_{g, 1} / \mathcal{M}_{g, 1}(k) \longrightarrow \mathrm{Sp}(2 g ; \mathbb{Z}) \longrightarrow 1
$$

where $\mathcal{I}_{g, 1} / \mathcal{M}_{g, 1}(k)$ turn out to be nilpotent groups. If we use here the affirmative solution by Hain [H2], to a conjecture made by the second author in [Mo5], which claims that the above extension splits over the rationals, then we can conclude that there exists a natural isomorphism

$$
\begin{aligned}
\lim _{g \rightarrow \infty} H_{c}^{*}\left(\mathcal{M}_{g, 1} ; \mathbb{Q}\right) \cong \\
\quad \lim _{g \rightarrow \infty}\left(H^{*}(\operatorname{Sp}(2 g ; \mathbb{Z}) ; \mathbb{Q}) \otimes \lim _{k \rightarrow \infty} H^{*}\left(\mathcal{I}_{g, 1} / \mathcal{M}_{g, 1}(k) ; \mathbb{Q}\right)^{\mathrm{Sp}}\right) .
\end{aligned}
$$

Borel $[\mathrm{B} 1][\mathrm{B} 2]$ proved $\lim _{g \rightarrow \infty} H^{*}(\operatorname{Sp}(2 g ; \mathbb{Z}) ; \mathbb{Q})=\mathbb{Q}\left[c_{1}, c_{3}, \ldots\right]$. Moreover, in view of a result of $[\mathrm{Mu}][\mathrm{Mo} 1][\mathrm{Mi}]$, we may choose $c_{2 i-1}$ so that it pulls back to $e_{2 i-1}$ under the natural homomorphism $\mathcal{M}_{g, 1} \rightarrow \operatorname{Sp}(2 g ; \mathbb{Z})$. On the other hand, Theorem 4 defines an element $b_{i} \in \lim _{k \rightarrow \infty} H^{2 i}\left(\mathcal{I}_{g, 1} /\right.$ $\left.\mathcal{M}_{g, 1}(k) ; \mathbb{Q}\right)^{\mathrm{Sp}}$ for all $i$ such that $b_{i}$ pulls back to $e_{i}$ on the mapping class group. With these terminologies, we have

Theorem 5. The stable continuous cohomology $\lim _{g \rightarrow \infty} H_{c}^{*}\left(\mathcal{M}_{g, 1} ; \mathbb{Q}\right)$ of $\mathcal{M}_{g, 1}$ contains the polynomial algebra $\mathbb{Q}\left[c_{1}, c_{3}, \ldots, b_{1}, b_{2}, \ldots\right]$. It follows that the natural homomorphism

$$
\rho_{\infty}^{*}: \lim _{g \rightarrow \infty} H_{c}^{*}\left(\mathcal{M}_{g, 1} ; \mathbb{Q}\right) \longrightarrow \lim _{g \rightarrow \infty} H^{*}\left(\mathcal{M}_{g, 1} ; \mathbb{Q}\right)
$$

from the continuous cohomology to the usual cohomology has a big kernel because it contains the ideal of this polynomial algebra generated by the elements $b_{i}-c_{i}$ for all odd $i$.

It seems to be reasonable to conjecture that the stable continuous cohomology of $\mathcal{M}_{g, 1}$, with respect to the filtration $\left\{\mathcal{M}_{g, 1}(k)\right\}_{k \geq 0}$, is isomorphic 
to the polynomial algebra given above. This theorem also shows that, for all odd $i$, the two canonical cocycles for $e_{i}$, namely one coming from $\operatorname{Sp}(2 g ; \mathbb{Z})$ and the other expressed as an Sp-invariant cocycle on the Torelli group, are never cohomologous to each other at any finite level $\mathcal{M}_{g, 1} / \mathcal{M}_{g, 1}(k)$. This fact is closely related to the secondary characteristic classes of surface bundles which were introduced by the second author in [Mo7].

Now in a recent paper [H2], Hain proved the following remarkable result. Namely if we fix a complex structure on the reference topological surface, then it induces a mixed Hodge structure (MHS for short) on $\lim _{k \rightarrow \infty} H^{*}\left(\mathcal{I}_{g, 1} / \mathcal{M}_{g, 1}(k) ; \mathbb{Q}\right)^{\mathrm{Sp}}$ and moreover the natural homomorphism

$$
\lim _{k \rightarrow \infty} H^{*}\left(\mathcal{I}_{g, 1} / \mathcal{M}_{g, 1}(k) ; \mathbb{Q}\right)^{\mathrm{Sp}} \longrightarrow H^{*}\left(\mathcal{M}_{g, 1} ; \mathbb{Q}\right)
$$

is a MHS morphism. It follows that, the induced homomorphism over $\mathbb{C}$ between their associated gradeds preserves the gradings and also that $\lim _{k \rightarrow \infty} H^{*}\left(\mathcal{I}_{g, 1} / \mathcal{M}_{g, 1}(k) ; \mathbb{C}\right)^{\mathrm{Sp}}$ is canonically isomorphic to the continuous cohomology of the graded Lie algebra

$$
\bigoplus_{k>0} \mathcal{M}_{g, 1}(k) / \mathcal{M}_{g, 1}(k+1)
$$

This graded Lie algebra can be described as follows. Let $\mathcal{L}=\oplus_{k=1}^{\infty} \mathcal{L}_{k}$ be the free graded Lie algebra generated by $H$ and let $\mathcal{H}=\oplus_{k=0}^{\infty} \mathcal{H}_{k}$ be the graded Lie algebra consisting of derivations of $\mathcal{L}$ which kill the symplectic class $\omega_{0} \in \Lambda^{2} H=\mathcal{L}_{2}$. $\mathcal{H}_{0}$ is nothing but the Lie algebra of $\operatorname{Sp}(2 g ; \mathbb{Q})$ and $\mathcal{H}_{k}$ for $k>0$ can be explicitly written as

$$
\mathcal{H}_{k}=\operatorname{Ker}\left(H \otimes \mathcal{L}_{k+1} \rightarrow \mathcal{L}_{k+2}\right)
$$

where the mapping $H \otimes \mathcal{L}_{k+1} \rightarrow \mathcal{L}_{k+2}$ is given by the bracket operation of the Lie algebra $\mathcal{L}$ (see [Mo6] for details). Now, for all $k \geq 1$, we have injective homomorphisms

$$
\tau_{k}: \mathcal{M}_{g, 1}(k) / \mathcal{M}_{g, 1}(k+1) \longrightarrow \mathcal{H}_{k}
$$

which are called Johnson homomorphisms (see [J2][Mo5][Mo6] for details). The direct sum $\operatorname{Im} \tau=\oplus_{k} \operatorname{Im} \tau_{k}$ can be naturally considered as a Lie subalgebra of $\mathcal{H}_{+}=\oplus_{k>0} \mathcal{H}_{k}$. Hain's result above can now be stated as an isomorphism

$$
\lim _{k \rightarrow \infty} H^{*}\left(\mathcal{I}_{g, 1} / \mathcal{M}_{g, 1}(k) ; \mathbb{C}\right)^{\mathrm{Sp}} \cong H_{c}^{*}(\operatorname{Im} \tau ; \mathbb{C})^{\mathrm{Sp}}
$$


where the right hand side denotes the Sp-invariant part of the continuous cohomology of the Lie algebra $\operatorname{Im} \tau$.

Now $\operatorname{Im} \tau$ is contained in $\mathcal{H}_{+}$by the definition and it is known that $\operatorname{Im} \tau_{1}=\mathcal{H}_{1}=\Lambda^{3} H$. Hence we have a series of homomorphisms each of which preserves the weight gradings

$$
H^{*}\left(\Lambda^{3} H ; \mathbb{C}\right)^{\mathrm{Sp}} \longrightarrow H_{c}^{*}\left(\mathcal{H}_{+} ; \mathbb{C}\right)^{\mathrm{Sp}} \longrightarrow H_{c}^{*}(\operatorname{Im} \tau ; \mathbb{C})^{\mathrm{Sp}} \longrightarrow H^{*}\left(\mathcal{M}_{g, 1} ; \mathbb{C}\right) .
$$

Kontsevich [Ko] described $\lim _{g \rightarrow \infty} H_{c}^{*}\left(\mathcal{H}_{+}\right)^{\text {Sp }}$ in terms of the cohomology of the outer automorphism groups Out $F_{n}$ of free groups $F_{n}$ of rank $n>1$. In particular he constructed homomorphisms

$$
H_{k}\left(\text { Out } F_{n} ; \mathbb{Q}\right) \longrightarrow \lim _{g \rightarrow \infty} H^{2 n-2-k}\left(\mathcal{M}_{g} ; \mathbb{Q}\right)
$$

for all $k$ and $n>1$ and asked the geometrical or "physical" meaning of these maps. Looijenga [L2] (see also [HL]) informed us the following argument which answers this question completely. He first applies the purity theorem of Pikaart $[\mathrm{P}]$, which says that the MHS of $H^{k}\left(\mathcal{M}_{g} ; \mathbb{C}\right)$ is pure of weight $k$ for $2 k+1 \leq g$, to conclude that the above map is the zero-map for all $k>0$. This is because the weight of any element of $H_{k}\left(\right.$ Out $\left.F_{n} ; \mathbb{Q}\right) \subset$ $\lim _{g \rightarrow \infty} H_{c}^{2 n-2-k}\left(\mathcal{H}_{+} ; \mathbb{Q}\right)$ is strictly bigger than the degree $2 n-2-k$. Then our main theorem, which determines the homomorphism $H^{*}\left(\Lambda^{3} H ; \mathbb{Q}\right)^{\mathrm{Sp}} \rightarrow$ $H^{*}\left(\mathcal{M}_{g, 1} ; \mathbb{Q}\right)$ completely, implies that it sends $1 \in H_{0}\left(\right.$ Out $\left.F_{n} ; \mathbb{Q}\right)$ to the class $e_{n-1} \in H^{2 n-2}\left(\mathcal{M}_{g} ; \mathbb{Q}\right)$. Thus the homology of various Out $F_{n}$ does not contribute to the stable cohomology of the mapping class group except for the degree zero parts where they correspond to the stable classes $e_{i}$.

The results of [Mo6] and [N] already show that the quotient $\mathcal{H}_{+} / \operatorname{Im} \tau$ is very big. In particular, the continuous cohomology of the Lie algebra $\operatorname{Im} \tau$ should be much closer to the cohomology of the moduli space than that of $\mathcal{H}_{+}$which was described by Kontsevich.

Now if we combine our main theorem with Hain's result [H2] together with Looijenga's argument as above, but applied to $\operatorname{Im} \tau$ rather than $\mathcal{H}_{+}$, then we can conclude

Theorem 6. The continuous part of the stable cohomology of the mapping class group, namely the image of the homomorphism $\rho_{\infty}^{*}: \lim _{g \rightarrow \infty} H_{c}^{*}\left(\mathcal{M}_{g, 1} ; \mathbb{Q}\right) \longrightarrow \lim _{g \rightarrow \infty} H^{*}\left(\mathcal{M}_{g, 1} ; \mathbb{Q}\right)$, is exactly equal to the subalgebra generated by the Mumford-Morita-Miller classes.

In the above discussions, we can also consider another filtration $\left\{\mathcal{M}_{g, 1}^{\prime}(k)\right\}_{k}$ on the mapping class group which is induced from the lower central series of the Torelli group. Using another fundamental result of Hain in [H1], we can obtain similar statements to Theorem 5 and Theorem 6 for the continuous cohomology associated with this filtration. However here we omit the details. 


\section{Acknowledgements}

The authors would like to express their gratitude to Richard Hain for many enlightening discussions and to Eduard Looijenga for useful informations and correspondences. They also would like to thank Hiroaki Nakamura and Makoto Matsumoto for discussions and comments.

\section{References}

[B1] A. Borel, Stable real cohomology of arithmetic groups, Ann. Sci. Ecole Norm. Sup. 7 (1974), 235-272.

[B2] Stable real cohomology of arithmetic groups II, Manifolds and Groups, Papers in Honor of Yozo Matsushima, Progress in Math., vol. 14, Birkhäuser, Boston, 1981, pp. 21-55.

[H1] R. Hain, Completions of the mapping class group and the cycle $C-C^{-}$, Mapping Class Groups and Moduli Spaces of Riemann Surfaces, Contemp. Math., vol. 150, 1993, pp. 75-105.

[H2] Infinitesimal presentation of the Torelli group, Preprint.

[HL] R. Hain and E. Looijenga, Mapping class groups and moduli spaces of curves, Preprint.

[Ha] J. Harer, Stability of the homology of the mapping class group of an orientable surface, Ann. of Math. 121 (1985), 215-249.

[HS] G. Hochschild and J. P. Serre, Cohomology of group extensions, Trans. Amer. Math. Soc. 74 (1953), 110-134.

[J1] D. Johnson, An abelian quotient of the mapping class group $\mathcal{I}_{g}$, Math. Ann. 249 (1980), 225-242.

[J2] _ A survey of the Torelli group, Contemporary Math. 20 (1983), 165179.

[Ka1] N. Kawazumi, A generalization of the Morita-Mumford classes to extended mapping class groups for surfaces, Preprint, Hokkaido Univ. (1995).

[Ka2] - On the stable cohomology algebra of extended mapping class groups for surfaces, Preprint, Hokkaido Univ. (1995).

[Ka3] - An infinitesimal approach to the stable cohomology of the moduli of Riemann surfaces, to appear in Topology and Teichmüller Spaces, World Scientific, Singapore.

[Ko] M. Kontsevich, Feynman diagrams and low-dimensional topology, Proceedings of the first European Congress of Mathematicians vol. 2, Progress in Math., vol. 120, Birkhäuser, Boston, 1994, pp. 97-121.

[L1] E. Looijenga, Stable cohomology of the mapping class group with symplectic coefficients and of the universal Abel-Jacobi map, J. Algebraic Geometry 5 (1996), 135-150.

[L2] private communication.

[Mi] E. Y. Miller, The homology of the mapping class group, J. Diff. Geom. 24 (1986), 1-14.

[Mo1] S. Morita, Characteristic classes of surface bundles, Invent. Math. 90 (1987), 551-577.

[Mo2] , Families of Jacobian manifolds and characteristic classes of surface bundles I, Ann. Inst. Fourier 39 (1989), 777-810. 
[Mo3] Families of Jacobian manifolds and characteristic classes of surface bundles II, Math. Proc. Camb. Phil. Soc. 105 (1989), 79-101.

[Mo4] - Mapping class groups of surfaces and three dimensional manifolds, Proc. Intern. Congress Math., Kyoto 1990, Springer, 1991, pp. 665-674.

[Mo5] - The extension of Johnson's homomorphism from the Torelli group to the mapping class group, Invent. Math. 111 (1993), 197-224.

[Mo6] Abelian quotients of subgroups of the mapping class group of surfaces, Duke Math. J. 70 (1993), 699-726.

[Mo7] _ Casson invariant, signature defect of framed manifolds and the secondary characteristic classes of surface bundles, Preprint.

[Mo8] A linear representation of the mapping class group of orientable surfaces and characteristic classes of surface bundles, to appear in Topology and Teichmüller Spaces, World Scientific, Singapore.

$[\mathrm{Mu}]$ D. Mumford, Towards an enumerative geometry of the moduli space of curves, Arithmetic and Geometry, Progress in Math., vol. 36, Birkhäuser, Boston, 1983, pp. 271-328.

[N] H. Nakamura, Coupling of universal monodromy representations of GaloisTeichmüller modular groups, Math. Ann. 304 (1996), 99-119.

[P] M. Pikaart, An orbifold partition of $\bar{M}_{g}^{n}$, The Moduli Space of Curves, Progress in Math., vol. 129, Birkhäuser,, Boston, 1995, pp. 467-482.

Departiment of M athematics, Faculty of Science, Hokkaido Univerity, S A P P ORO, 060 JAPA N

E-mail address: kawazumi@math.hokudai.ac.jp

Department of Mathematical Sciences, Univeriti of Tokyo, Tokyo, 153 J A P A N

E-mail address: morita@ms.u-tokyo.ac.jp 